\title{
Mental state
}

\section{David F Marks}

Potential competing interests: The author(s) declared that no potential competing interests exist.

A mental state is a condition of an agent that includes purpose, desire and intention. 